\title{
« Renouvelez-vous pour moi, moments tranquilles de ma douce adolescence! » Sur les moments d'otium dans la Révolution française
}

About Moments of Otium during the French Revolution

\section{Franziska Meier}

\section{OpenEdition}

Journals

Édition électronique

URL : https://journals.openedition.org/recherchestravaux/800

DOI : 10.4000/recherchestravaux.800

ISSN : 1969-6434

Éditeur

UGA Éditions/Université Grenoble Alpes

Édition imprimée

Date de publication : 1 juin 2016

Pagination : 15-30

ISBN : 978-2-84310-325-4

ISSN : 0151-1874

\section{Référence électronique}

Franziska Meier, « «Renouvelez-vous pour moi, moments tranquilles de ma douce adolescence! » Sur les moments d'otium dans la Révolution française ", Recherches \& Travaux [En ligne], 88 | 2016, mis en ligne le 01 janvier 2017, consulté le 29 octobre 2021. URL : http://journals.openedition.org/

recherchestravaux/800; DOI : https://doi.org/10.4000/recherchestravaux.800 
Franziska MeIer

Université Georgia Augusta de Göttingen

\section{«Renouvelez-vous pour moi, moments tranquilles de ma douce adolescence!» Sur les moments d'otium dans la Révolution française}

Parler d'otium au moment et à propos de la Révolution française semble presque faire contresens. Toutes les images mentales qui se présentent à nous pour peu que l'on pense à la dernière décennie du XviII ${ }^{e}$ siècle français sont marquées par ce mouvement d'enthousiasme effréné, de violence, de joie et de douleur, enfin par ce que le XVIII ${ }^{\mathrm{e}}$ siècle a reconnu - comme le dit Michel Delon - sous le terme «énergie ${ }^{\mathrm{I}}$ ». Cette impression générale est confirmée par les contemporains de la Révolution, aussi bien ses adversaires que ses partisans qui, à leur grande surprise, se voyaient happés par une dynamique qu'ils avaient euxmêmes délibérément déclenchée en 1789 et qu'ils avaient de plus en plus de mal à maîtriser. Bon gré mal gré, ils étaient entraînés d'une péripétie à l'autre, d'un extrême à l'autre, sans avoir jamais la possibilité de reprendre haleine et d'exercer un contrôle sur un mouvement devenu irrésistible. On a comparé à juste titre la Révolution à une catastrophe naturelle, à un torrent qui sort de son lit et bouleverse les rives aux alentours sans laisser ni refuges ni îles de repos ${ }^{2}$.

Répit impossible, moments de loisir interdits : cette cadence infernale trouve un symbole très visible dans le fait que la Révolution a transformé un des hauts lieux de loisir situés à Versailles en un lieu éminemment politique. C'est au Jeu de Paume, construit au XVII ${ }^{\mathrm{e}}$ siècle, que le 20 juin $\mathrm{I} 789$ une grande

I. Voir M. Delon, L'Idée d'énergie au tournant des Lumières (I770-I820), Paris, PUF, 1988.

2. Voir à ce propos H. Arendt, On Revolution, New York, Viking Press, 1963, et R. Koselleck, Vergangene Zukunft. Zur Semantik geschichtlicher Zeiten, Francfort-sur-le-Main, Suhrkamp, 1979. 
partie des députés du tiers état et quelques-uns de ceux du clergé et de la noblesse ont échangé des serments solennels. En réponse à l'ordre du roi de dissoudre la réunion des états généraux, ils se jurèrent mutuellement de ne pas se séparer avant d'avoir élaboré et donné une constitution à la France. Sur le plan juridique et politique, les conséquences de ce serment revêtent une tout autre importance que la prise de la Bastille. La Révolution a été décidée dans un lieu de loisir, pour ainsi dire, où l'aristocratie de cour, apprivoisée par le monarque absolu, avait coutume de prendre du bon temps.

On pourrait retenir le nom d'un témoin très surprenant, mais significatif de la manière dont l'individu a vécu le déclenchement de cette dynamique révolutionnaire : Rétif de la Bretonne. Inspiré et encouragé par le succès du Tableau de Paris que Louis-Sébastien Mercier avait commencé à publier en I78I, Rétif pensait profiter de ce nouvel engouement des lecteurs. Il ambitionnait de proposer au public français une description du Paris nocturne, dans laquelle il faisait étalage de ses vaillantes excursions dans les zones interlopes de la capitale, dans un ouvrage intitulé Les Nuits de Paris. Les premiers volumes parurent en I788, peu avant la Révolution. À la différence de Mercier qui ne relancera qu'après la Terreur son projet d'un tableau de Paris sous le titre Le Nouveau Paris, Rétif, lui, n'interrompt pas sa production et sa publication de nouvelles en I789. Son alter ego, un personnage appelé "hibou», continue de vivre et d'observer la vie à Paris durant les grands événements de la Révolution. L'année suivante, Rétif publie un septième volume qui a pour titre La Semaine nocturne, que suivra un huitième volet, Vingt Nuits de Paris, en I794. Ces deux ouvrages, qui ont été réunis par Béatrice Didier sous le titre Les Nuits révolutionnaires ${ }^{3}$, reflètent les impressions de Rétif et révèlent ce qui lui est arrivé entre 1789 et I793 - s'il faut en croire ses protestations de sincérité.

Au début, Rétif semblait mettre hors de doute le fait qu'il pouvait simplement continuer sa série de nouvelles. Cependant, alors que la Révolution se déploie, il prend peu à peu conscience de l'ampleur des bouleversements qu'elle a entraînés dans l'État français, dans la société et ce jusque dans les détails de la vie quotidienne. Les recherches $\mathrm{du}$ «hibou» ne se limitent plus aux heures nocturnes; bien au contraire, la nuit semble avoir étendu son règne. Au lendemain de la prise de la Bastille, le monde du crime, du vice et de la prostitution n'a manifestement plus de honte à se montrer au grand jour ${ }^{4}$. Très vite, le «hibou »

3. N. Rétif de la Bretonne, Les Nuits révolutionnaires, éd. B. Didier, Paris, Le Livre de Poche, 1978. (Désormais $N R$.)

4. Voir D. Masseau : "Ce qui n'était dans les Nuits de Paris qu'une enquête pittoresque sur les illégalismes parisiens devient la mise à jour d'un enfer sexuel qui symbolise les débordements de la Révolution tout entière." ("L'irruption de l'histoire dans l'écriture des Nuits révolutionnaires", Revue des Sciences humaines, ${ }^{\circ}$ 212, 1988, p. 37.) 
doit s'avouer qu'il n'est plus capable de débusquer et de combattre le crime comme auparavant. Désormais la violence populaire permet à la débauche de se répandre en tous lieux, et, sous couvert de la liberté proclamée, de s'imposer à l'innocence que le «hibou» ne réussit plus à préserver. Dans les rares cas où il réussit à combattre le mal, il est assisté par d'autres bourgeois et artisans qui, tout comme lui, souhaitent à tout prix maintenir l'ordres. Dès lors, il apparaît clairement que ce n'est plus Rétif qui part à la recherche des zones d'ombre de Paris pour dénoncer quelques "affaires particulières", mais que ce sont plutôt les affaires politiques et les événements qui viennent, si l'on peut dire, le chercher chez lui et l'arracher à ses devoirs d'éditeur et d'imprimeur, et à sa vie privée ${ }^{6}$. En effet, lorsqu'on lit le premier recueil des Nuits révolutionnaires, on s'aperçoit que le personnage du "hibou» est pris d'un mouvement sans relâche, qui ne lui concède plus aucune autonomie. La description suivante semble particulièrement éloquente à ce propos :

J'entendais un mouvement sourd; je voyais des gens marcher isolés, mais à peu de distance : je sentais au-dedans de moi un mouvement tumultueux; il semblait que l'agitation de ceux qui fuyaient m'électrisât [...]. Le physique quelquefois dans l'homme remplacerait-il le moral? (NR, p. 2I2)

De son cabinet de travail, le «hibou» de Rétif entend des rumeurs qu’il associe immédiatement à un mouvement. Il voit ensuite des gens passer dans la rue et qui semblent poussés par une force imperceptible à se rassembler de façon à ne former qu'une seule et unique masse, celle d'un peuple révolté. Cette impulsion se transmet immédiatement au spectateur Rétif qui, quoiqu'occupé à autre chose, n'est pas capable de résister. Le mouvement extérieur paraît s'emparer de ses sens. Le narrateur compare l'effet produit à une "décharge électrique", soit à un phénomène irrépressible, de sorte qu'un semblable mouvement "tumultueux" se reproduit en lui. Le "hibou» finit par rejoindre la foule et par marcher, ignorant tout comme elle le pourquoi et le but de ce cortège. Il a malgré tout le pressentiment qu'elle le mènera tout droit à l'une des journées de la Révolution.

Ce changement de fond ne concerne pas seulement le personnage fictif. Il est étonnant de constater à quel point il atteint même la composition et le style. En premier lieu, Rétif doit abandonner sa stratégie qui consiste à faire

5. On en trouve une occurrence dans $N R$, p. 335.

6. Voir J. Lecuru : «Dans les NdP le Hibou créait l'événement en rendant publics les drames particuliers et les dessous mystérieux des mœurs nocturnes. À partir de 1789 c'est l'Histoire qui se charge, avec rapidité et tumulte, de multiplier les événements. Le domaine public l'emporte sur le domaine privé, mais la tradition intertextuelle héritée des 380 nuits déjà publiées oblige Rétif à ne pas sacrifier le second domaine au premier.» ("Le hibou en révolution ou du spectateur nocturne au citoyen spectateur nocturne», Revue des Sciences humaines, $\mathrm{n}^{\circ}$ 2I2, I988, p. 39.) 
alterner ses incursions dans les milieux louches et criminels de Paris avec des excursions nouvelles, paisibles et sans troubles, pour ne pas dire idylliques. En effet, ce mouvement qui l'entraîne l'empêche en même temps de créer un espace à l'abri dans son recueil. Bref, c'est une agitation qui déteint sur la composition elle-même. Plus révélateur encore, l'écrivain avertit dans l'Avis aux lecteurs des Vingt Nuits de Paris qu'en dépit d'un récit "à la première personne, pour ne pas varier mal à propos le style» ( $N R$, p. 190), le pronom «je» ne se réfère plus à ce qu'il a vu et vécu personnellement. Derrière ce «je» se cachent d'autres observateurs de la Révolution en qui il a pleine confiance. Ce mélange des identités regroupées sous un seul «je» s'explique par le fait que, sous la Révolution, la description de la vie parisienne est devenue trop ardue et réclame davantage que les forces d'un seul auteur. Rétif admet son impuissance à couvrir un tel projet et, ce faisant, semble sur le point d'abdiquer son autorité souveraine d'auctor. Enfin, ce mouvement irrésistible semble s'introduire jusque dans le style de Rétif, certes depuis toujours plein d'allant et caractérisé par une certaine vivacité, mais qui, au fur et à mesure de la rédaction des Nuits révolutionnaires, se fait de plus en plus haché, de plus en plus confus. La ponctuation parle d'elle-même : les points d'exclamation ainsi que les points de suspension s'enchaînent, et les questions en cascade, les signes d'angoisse et d'incertitude insérés dans le texte se multiplient. Les dernières "additions» enfin que Rétif ajoute pendant les mois de la Terreur - notamment sa profession de foi montagnarde -, affichent une langue délirante, qui ne fait que répéter à outrance les nouveaux termes, empruntés au latin, de la Révolution et superposer des aperçus à la hâte ${ }^{7}$.

Il est intéressant de noter que Rétif, après la chute de Robespierre et du Comité de salut public, ne s'est pas décidé à revoir son style. Selon ses dires, "ce vernis, parce qu'il est historique autant que la narration elle-même" $(N R$, p. 190) lui tient à cœur; c'est une preuve de sa sincérité. À son avis, c'est par son style - trop négligé du point de vue de la rhétorique classique - que se comprennent le mieux les conditions d'existence d'un contemporain de la Révolution et les contraintes auxquelles est soumis un auteur «victime» du mouvement révolutionnaire en marche. L'élargissement du je narré à d'autres observateurs ainsi que l'impossibilité de toute retraite vers un intérieur protégé dont se plaint occasionnellement le "hibou» correspondent en cela à la politique menée sous la Terreur, qui visait la transparence absolue au sein de la société, et qui en conséquence rendait suspect tout désir d'une vie privée, et partant tout moment de repos et de loisir. Les Nuits de Paris du temps de la Révolution font certainement figure de cas extrême et vraisemblablement

7. Voir $N R$, p. 385-388. 
exagéré - tout autant que l'était la position de Rétif dans le panorama littéraire de son époque ${ }^{8}$. Peut-on néanmoins affirmer que cette réaction littéraire à la Révolution, suscitée par l'invasion du mouvement irrésistible au cœur de la vie du sujet et dans son écriture, n'est qu'un phénomène isolé?

L'impact de la dynamique révolutionnaire ne se résume certainement pas à l'effet produit sur Rétif, pas plus qu'il ne se limite à ceux qui y adhèrent avec enthousiasme, ou qu'il ne s'arrête aux frontières de la France. Cette dynamique parcourt en effet presque tous les écrits des émigrés et des proscrits qui, malgré la distance, ne parviennent pas à détacher leurs yeux des événements qui se précipitent en France, ni à prendre du recul par rapport au mouvement enclenché. Le jeune Chateaubriand par exemple, émigré en Angleterre, développe dans son Essai sur les révolutions une argumentation pertinente sur le caractère inéluctable de ce mouvement révolutionnaire. Il cherche à expliquer la Révolution au moyen d'une comparaison avec les révolutions anciennes, issues de l'histoire grecque pour la plupart, espérant ainsi qu'elle s'achève, conformément à la théorie des cycles de Polybe qui veut que l'histoire revienne toujours au point de départ, c'est-à-dire, en l'occurrence, à la monarchie. À force d'élaborer les différentes étapes qui constituent un cycle - soit l'équivalent du premier sens donné au mot "révolution" -, Chateaubriand établit une sorte de succession chronologique de cycles dont le fond négatif va s'accroissant et qui culmine avec la Révolution française. Désespéré de pouvoir un jour enrayer la dynamique, le jeune émigré finit par figer la dernière Révolution dans une espèce de paroxysme qui ne peut aboutir quà deux solutions également démesurées : l'entrée dans une sorte de paradis ou le retour à la barbarie. Le cas de Chateaubriand suffit pour l'instant, à lui seul, à prouver que la Révolution française s'infiltre progressivement dans la façon de penser et de vivre de ceux qui l'ont vécue, et à montrer de quelle manière se fait cette intrusion; acteurs ou spectateurs, de bon ou de mauvais gré, tous l'ont constaté'. Compte tenu de l'omniprésence de la Révolution, peut-on raisonnablement parler de moments d'otium - même dans le sens le plus vertueux de «solitude lettrée» - dans un contexte en soi incongru, et qui est si manifestement défavorable à l'otium? Étudier ses liens avec l'écriture dans la littérature française de cette époque-là a-t-il un sens?

Du point de vue de l'histoire littéraire, la littérature de la période révolutionnaire est à considérer comme étant à la marge du cours de l'Histoire,

8. Voir P. Hartmann, Rétif de la Bretonne entre individu et communauté, Paris, Desjonquères, 2009.

9. Je renvoie à mes analyses dans la première partie de mon livre In ein Mühlwerk geworfen. Zum autobiographischen Schreiben in der Französischen Revolution, Göttingen, Vandenhoeck \& Ruprecht, 20I6. 
discordante, comme à part, détachée à la fois de la tournure que prenaient les événements et de leur répercussion immédiate et étonnante sur les hommes et les femmes d'alors. Vue sous cet angle, la seule idée qu'une dynamique politique puisse avoir atteint l'écriture d'une époque sonne faux, l'hypothèse d'une influence de la première sur la seconde paraît presque absurde. Ainsi, jusqu'au bicentenaire de la Révolution française, reprocher à la littérature de la dernière décennie du XVIII ${ }^{\mathrm{e}}$ siècle d'avoir manqué à l'appel de 1789 était monnaie courante. Cette littérature était dépréciée pour être restée fidèle aux genres traditionnels et à la poétique du classicisme sans pouvoir rivaliser avec le brillant succès obtenu par le peintre Jacques-Louis David. La critique littéraire moderne, en cela, avait hérité de la déception exprimée par Victor Hugo, qui retardait au 25 février I830 - à savoir à la bataille d'Hernani - l'entrée en scène des Jacobins et dans le même temps la conversion tant attendue du champ littéraire. On doit aux travaux de Béatrice Didier et de Michel Delon (pour la critique française), de Fritz Nies et de Hans Ulrich Gumbrecht (pour la critique allemande) d'avoir attiré l'attention sur le nombre et la forme des nouveaux genres qui émergent à la Révolution, en insistant sur les innovations proposées par la pratique du mélange des genres en rupture avec la poétique classique, et d'avoir ainsi sorti de l'oubli une production littéraire jusque-là mésestimée ${ }^{\mathrm{I}}$. Précisons toutefois que ces tentatives n'ont jamais eu pour objectif de remettre en question la suprématie sans conteste du système classiciste. Il est donc très probable que dans le champ littéraire, en dépit des changements apportés par la Révolution, les auteurs continuaient de considérer la littérature comme un lieu de retraite et, par conséquent, qu'ils avaient conservé ce qu'on pourrait appeler les «attentes traditionnelles» vis-à-vis de l'écriture.

Comment faut-il comprendre exactement l'expression «attentes traditionnelles» vis-à-vis de l'écriture, et comment appréhender leur lien avec le concept d'otium? À ce sujet, il convient de faire appel, au moins de manière succincte,

IO. Voir B. Didier, Écrire la révolution, I789-I799, Paris, PUF, 1989, et M. Delon, «La Révolution et le passage des Belles-lettres à la littérature ", Revue d'histoire littéraire de la France, nº 90, 1990, p. 573-588 et, plus récemment, I. Brouard-Arends et L. Loty (dir.), Littérature et engagement pendant la Révolution française, Rennes, PUR, 2007, et A. Déruelle et J.-M. Roulin (dir.), Les Romans de la révolution, I790-I9I2, Paris, Armand Colin, coll. "Recherches», 20I4. Du côté allemand, voir F. Nies, "Das System der literarischen Gattungen. Kontinuitäten, Brüche, Schwerpunktzonen (1789-I799)", dans R. Koselleck et R. Reichardt (dir.), Die Französische Revolution als Bruch des gesellschaftlichen Bewußtseins, Munich, Oldenbourg, I988, p. 243-275, et H. U. Gumbrecht, «Skizze einer Literaturgeschichte der Französischen Revolution », dans J. von Stackelberg (dir.), Neues Handbuch der Literaturwissenschaft / Europäische Aufklärung, t. I3, Wiesbaden, Athenaion, I980, p. 269-328. 
aux remarques de Michel Foucault dans son Herméneutique du sujet ${ }^{\text {II }}$. Il y traite de la notion d'epimeleia heautou (souci de soi), omniprésente dans la pensée antique, qui l'avait fasciné très tôt dans sa carrière. Selon Foucault, pour la philosophie antique, le souci de soi s'inscrit pour le sujet dans tout processus de recherche de la vérité et l'anticipe même, requérant une certaine attitude, une attention scrupuleuse sur ce que l'on fait, sur ce que l'on ressent et sur ce que l'on pense, prenant appui sur des techniques spécifiques de méditation et d'autoréflexion. Et c'est ici que l'écriture entre en jeu. Son rôle essentiel est d'organiser les idées, de contrôler les sensations et les émotions en les extériorisant «sur le papier» et, ce faisant, de (re)trouver l'équilibre. Par le truchement de l'écriture qui dans l'Antiquité s'adressait autant au sujet lui-même qu'à un autre - notamment à un ami -, le sujet prend du recul et se crée un espace de liberté en dehors des contraintes sociales et mondaines, où il peut s'épanouir et se recueillir. L'écriture pour cette raison est profondément liée à l'espace d'otium au sens où Sénèque l'entendait : elle en est à la fois l'une des expressions et l'instrument privilégié.

À quelques changements et déformations près, de nombreuses facettes de cet héritage majoritairement stoïcien ont survécu à la chute de l'empire romain et, au cours des siècles, se sont mêlées aux pratiques du christianisme, qui s'en était inspiré pour sa part. On les retrouve chez les humanistes, et aussi un peu plus tard, d'abord chez Montaigne, lorsqu'il se met en retrait des «negoces» de sa vie pour se retirer à la campagne dans une atmosphère propice à l'otium, se vouant ainsi à l'écriture, à des méditations au long cours dans lesquelles alternent lectures et réflexions, et ensuite chez Descartes, par exemple, dont la philosophie prend son essor dans une retraite volontaire et absolue, sorte d'antichambre nécessaire à un espace d'otium, mais pour permettre cette fois au philosophe de reconstruire sa propre vision du monde à partir de ce qui s'est révélé à lui comme certain, et en mettant en jeu sa subjectivité stricto sensu.

En poursuivant la chronologie, il n'y a aucun doute que l'héritage latin, le rapport entre otium et écriture, est encore bien présent au XVIII ${ }^{\mathrm{e}}$ siècle. Si l'on s'en tient aux Rêveries du promeneur solitaire, Rousseau ne s'est pas contenté d'adopter la notion latine (et ses excroissances successives), mais se l'est appropriée d'une manière très originale en donnant à l'écriture une valeur jusquelà inouïe. En effet, dans les Rêveries l'écriture a un rôle encore plus décisif, puisqu'elle est censée pérenniser les moments de bonheur que Jean-Jacques se remémore, afin qu'il puisse, à un âge plus avancé, les retrouver lui-même, dans leur fraîcheur fragile, grâce à la lecture. Chez Rousseau, l'écriture ne se limite

\section{L'Herméneutique du sujet : Cours au Collège de France (I981-I982), Paris, Seuil, 2001.}


donc pas à servir d'instrument pour extérioriser et remettre en ordre les idées et les émotions. Elle est devenue une sorte de réservoir, ou de "congélateur» pourrait-on dire aujourd'hui, destiné à recueillir et à conserver dans toute leur vivacité des sensations particulières, sources d'épanouissement personnel qui servent de points de repère à l'individu dans la recherche de sa vérité subjective ${ }^{12}$. En somme, à l'image de Rousseau, le XviII siècle semble avoir gardé et même renforcé - dans la seconde moitié du siècle - le concept stoïcien de l'écriture de soi comme exercice spirituel. Cependant, étant donné que la dynamique révolutionnaire a assurément eu des répercussions fortes sur l'intériorité du sujet, il faut se demander dans quelle mesure et comment les auteurs de l'époque ont pu préserver l'héritage latin, dans sa forme d'origine ou dans la variation proposée par Rousseau, et si cette technique de retour à soi leur a fourni un dernier rempart contre la vague qui menaçait de les submerger ${ }^{13}$.

Les textes autobiographiques de l'époque se prêtent, me semble-t-il, à une réponse possible à ces questions. Le genre des mémoires était alors très en vogue : le corpus est vaste et ses formes sont quelque peu hétérogènes. Il embrasse tout un panel d'écrits, du petit morceau de papier griffonné par les prisonniers sous la Terreur, coupés de tout contact avec l'extérieur, qui souhaitaient faire parvenir un signe ou un dernier vœu à leurs proches, aux mémoires plus ou moins volumineux écrits pour la plupart à la même période dans les prisons ou dans des cachettes, à Paris ou en province. Ces récits ont donc une double ambition : raconter l'histoire d'une vie et/ou plaider politiquement sa cause. Plusieurs mémoires naissent sous la plume des Girondins qui, contrairement aux Jacobins-Montagnards, ont disposé de quelques mois de loisir qu' ils pouvaient consacrer à une entreprise d'une telle envergure entre le moment de leur proscription ou arrestation et le jour de leur exécution. Ces mémoires sont bien évidemment les témoignages les plus élaborés, et en tant que tels apparaissent à mon avis comme le terrain le plus représentatif et le plus favorable à un examen pour comprendre les rapports entre otium et écriture à la fin du XviII ${ }^{\mathrm{e}}$ siècle.

Tous ces mémorialistes, quelle que soit leur opinion politique, s'engagent à écrire leur vie dans un moment où elle est sérieusement mise en péril. Volontaire

I2. Je renvoie à titre d'exemple à ce que Rousseau dit de son projet dans la première promenade des Rêveries : "Les loisirs de mes promenades journalières ont souvent été remplis de contemplations charmantes dont j'ai regret d'avoir perdu le souvenir. Je fixerai par l'écriture celles qui pourront me venir encore; chaque fois que je les relirai m'en rendra la jouissance.» (J.-J. Rousseau, Les Rêveries du promeneur solitaire, éd. H. Roddier, Paris, Garnier, I960, p. 9.)

I3. Tout l'article reprend une argumentation beaucoup plus développée que j'ai menée dans mon livre In ein Mühlwerk geworfen. Zum autobiographischen Schreiben in der Französischen Revolution, ouvr. cité. 
ou forcée par les circonstances, la retraite solitaire dans laquelle ils sont plongés rappelle par certains aspects l'espace d'otium imposé tel que Rousseau l'a évoqué dans ses écrits autobiographiques. De fait, beaucoup des mémorialistes, avant tout parmi les Girondins, étaient conscients de l'analogie de leur situation avec celle des penseurs antiques, et s'empressaient de mettre à profit un tel état des choses pour appliquer à leur vie les préceptes stoïciens, en une sorte de pied de nez au destin et à l'adversité. Toutefois, aucun d'entre eux ne réussit à oublier la contrainte qui pesait sur sa vie, ni la précarité de son sort. Ils considéraient qu'ils étaient "condamnés à l'otium», pour reformuler une célèbre expression de Jean-Paul Sartre. L'assimilation de la situation des mémorialistes à celle traditionnellement associée à l'otium est ambiguë; on y trouve des ressemblances, mais on sent qu'ils s'inscrivent en porte-à-faux. D'un côté, ils suivent le modèle antique et s'efforcent de goûter les charmes et les bienfaits de leur temps de loisir forcé. Ils se servent en conséquence de l'écriture comme d'un moyen pour accéder à l'autoréflexion qui les aide à prendre du recul par rapport au tourbillon de la Révolution et, comme d'un lieu de recueillement, de retour à soi et à un sentiment de plénitude de l'existence. De l'autre, ils n'oublient pas de profiter de leur retraite imposée pour écrire des mémoires, dans un sens juridique cette fois, et, par là, préparer leur retour victorieux sur la scène politique. En d'autres termes, pour eux, l'écriture - dont les Lumières avaient souligné le pouvoir d'action - devient une arme politique, qu'on brandit aux yeux des Français même dans l'ombre de sa cachette ou de sa cellule de prison.

À titre d'exemples, je voudrais étudier de plus près des mémoires dont les auteurs diffèrent par leur âge, leur sexe, leur origine sociale, leur métier et leur engagement dans la Révolution : il s'agit de ceux de Jean-François Marmontel, et de ceux de Manon Roland. Le premier avait soixante-six ans en I789, et était connu en Europe pour être l'auteur de Contes moraux; la seconde était l'égérie de la faction girondine, et avait trente-cinq ans l'année de la Révolution. Alors que Marmontel avait rapidement pris ses distances avec la Révolution, rejoignant le groupe réactionnaire de farouches opposants réunis autour de l'abbé Maury, Madame Roland, elle, s'était rangée du côté de la Révolution dès la prise de la Bastille et avait prôné très tôt l'idée d'une constitution républicaine. Son mari, Roland de la Platière, ministre de l'Intérieur de mars 1792 à janvier I793, avait su tirer parti de l'habileté de plume et de la détermination de sa jeune épouse. Dès l'été I792, Marmontel, entraîné par sa famille proche, s'enfuit en Normandie et commence à remplir d'activités d'écriture ses longues journées de solitude. Madame Roland, en revanche, reste sur le théâtre de la Révolution à Paris, et sera arrêtée à la place de son mari le $\mathrm{I}^{\mathrm{er}}$ juin I793. Elle passe les cinq mois suivants - à l'exception d'une brève interruption - dans les prisons instaurées par la Terreur. Fière d'être jugée avec le même traitement 
que ses compagnons politiques masculins, elle meurt sous la guillotine le 8 novembre de la même année. Son amour de la liberté, le courage dont elle fait preuve dans sa marche vers la mort, mais aussi son charme n'auront de cesse d'émerveiller les écrivains et les historiens au XIX ${ }^{\mathrm{e}}$ siècle et de nourrir leur imagination.

Marmontel donne pour titre Mémoires d'un père pour servir à l'instruction de ses enfants à l'histoire de sa vie que sa veuve publiera en I805. Il est étonnant qu'un homme qui avait ébauché une théorie sur le genre littéraire des mémoires se détache aussi clairement, et de la tradition des mémoires de l'Ancien Régime écrits pour servir l'Histoire, et de la définition qu'il en avait donnée ${ }^{14}$. De fait, il présente son livre (assez volumineux) comme un écrit absolument privé qui ne s'adresse qu'à ses enfants. Dans le premier paragraphe, on peut lire ceci :

C'est pour mes enfants que j'écris l'histoire de ma vie; leur mère l'a voulu. ( $\mathrm{Si}$ quelque autre y jette les yeux, qu'il me pardonne les détails minutieux pour lui, mais je crois intéressants pour eux.) Mes enfants ont besoin de recueillir les leçons que le temps, l'occasion, l'exemple, les situations diverses par où j'ai passé m’ont données. Je veux qu'ils apprennent de moi à ne jamais désespérer d'eux-mêmes, mais à s'en défier toujours, à craindre les écueils de la bonne fortune, et à passer avec courage les détroits de l'adversitér ${ }^{15}$.

Dans ce passage le je narrant semble s'être retiré du monde, dans un cercle restreint à la famille. Il ne dit rien de leur retraite quelque part à la campagne; il précisera seulement, au paragraphe suivant, le nom du village. La solitude et son rôle de patriarche l'ont incité à raconter sa vie à ses enfants. À en juger d'après l'extrait précédemment cité, le récit de ses aventures est entièrement subordonné à des fins éducatives. Sa vie ne trouve sa raison d'être que dans la mesure où elle offre des leçons morales à transmettre aux jeunes gens. D'ailleurs, les grands changements que la Révolution apporte ne semblent pas avoir diminué la valeur exemplaire de ses expériences faites sous l'Ancien Régime. Dans la morale que l'on trouve à l'ouverture du récit, la Révolution apparaît certes, mais de manière implicite, et en outre sous une forme assez généralisée et abstraite, comme si Marmontel s'excluait du temps présent, comme s'il vivait en somme une existence qui répond par bien des aspects à la définition la plus aboutie de l'otium.

En parcourant le texte de Marmontel, on constate que les cinq cent soixante premières pages offrent un récit riche et détaillé qui applique à la lettre la

I4. Voir à ce propos les remarques de M. Cardy, "Marmontel's Mémoires Revisited», British Journal for Eighteenth-Century Studies, n ${ }^{17}$, 2, 1994, p. I8I.

I5. Mémoires, éd. J.-P. Guicciardi et G. Thierriat, Paris, Mercure de France, 1999, p. 49. (Désormais $M M$.) 
«recette» préconisée par Horace : plaire et instruire tout à la fois. La narration adopte le point de vue du je narré et ne s'interrompt qu'à l'occasion de quelques commentaires du je narrant, qui d'ailleurs assume une grande autorité. À la fin du livre XI pourtant, le récit s'arrête de façon inopinée. Il est vrai que le je narrant avait annoncé quelques pages plus tôt les malheurs à venir pour sa famille. Mais rien ne laissait présager la rupture qui sépare nettement le livre XI du livre XII. Elle est si éclatante que les deux éditeurs, Jean-Pierre Guicciardi et Gilles Thierriat, ont divisé le texte en deux parties distinctes bien que le manuscrit poursuive la division en livres numérotés.

Dans la conclusion du livre XI, Marmontel s'adresse directement au lecteur en abandonnant le ton enjoué et décidé pour lequel il avait opté jusque-là, pour expliquer que dès lors «les intérêts de la chose publique et les inquiétudes sur le sort de l'État s'emparèrent de [ses] esprits» et que sa vie privée changea de face, et prit une couleur qui nécessairement allait se répandre sur le reste de ses Mémoires (MM, p. 564). Marmontel insiste sur le changement survenu qu'il définit en des termes qui rappellent la distinction latine entre otium et negotium. Conscient de son devoir de citoyen, Marmontel - entendu comme le je narré des Mémoires - s'était détourné de son train de vie d'auteur, d'homme de lettres à la mode, et de sa vie mondaine pour se consacrer à la res publica. Cependant, au lieu du récit attendu de son engagement politique, le je narré disparaît sans même figurer comme spectateur privilégié des événements. C’est seulement au moment où le récit fait état de la convocation des états généraux que la narration autobiographique cède la place à une analyse et à une réflexion historiographique visant à faire comprendre les causes et les effets de 1789 et de la chute de la monarchie. L'auteur assume ici la charge d'historiographe royal que lui avait assignée Louis XV et que la Révolution avait abolie.

Le mémorialiste lui-même semble mal à l'aise avec ce changement qui se produit dans sa vie, réellement vécue ou transposée dans l'écrit. En effet, le livre XII s'ouvre sur l'affirmation suivante : "Je n'écris pas l'histoire de la Révolution» $(M M$, p. 567). Le récit respecte-t-il pour autant cette dénégation? Bien que le dernier tiers des Mémoires ne soit pas à proprement parler un récit d'événements et d'actions, il rapporte précisément ce que l'auteur entendait éviter : une histoire de la Révolution. Quelque soin que Marmontel mette à cacher qu'il ne contrôle désormais plus son texte - il entame une longue argumentation sur les fautes commises par le parti royaliste ainsi que sur les intentions criminelles d'un petit groupe de conjurés -, on sent bien, au moment où il répète "Ces Mémoires ne sont point l'histoire de la Révolution " $(M M$, p. 724$)$, qu'on ne peut plus croire à une quelconque maitrise de son récit. La déroute d'une pensée d'abord pleine d'autorité est encore plus flagrante dans la suite où son argumentation s'étiole à mesure que la Révolution 
se radicalise. Marmontel passe malgré lui d'une réflexion philosophique à une narration chronologique pauvre en faits historiques. Lorsqu'enfin les images qui viennent confirmer la chute de la famille royale parcourent toute la France à travers la presse et les brochures, l'illusion d'une idyllique retraite familiale à Habloville s'effondre définitivement. Le je narré disparaît derrière le nous de la famille qui tombe sous le choc de la nouvelle.

À la toute fin du volume, Marmontel laisse percevoir à quel point la Révolution l'a marqué. Dans le vingtième et dernier chapitre, il fait l'aveu de la détresse que lui inspire sa situation. Il en résulte que les années passées à Habloville n’ont rien à voir avec l'idéal d'otium. Le retrait de la vie publique n'a pas épargné au sujet les bouleversements extérieurs, et le laisse mentalement affaibli et sans ressources. Au final, le grand «récit de vie» promis au lecteur se révèle être une tentative désespérée de camoufler, sous le couvert d'une autoréflexion à visée propédeutique, ce qui n'est au fond que la manifestation d'une angoisse bien présente. Dans son désarroi, le je narrant se sert de l'écriture, si l'on veut, pour proposer une espèce de variation bourgeoise du concept latin, mais celle-ci s'avère bancale et n'est autre qu'un prétexte. La figure d'autorité du précepteur moral incontesté disparaît en même temps que le masque se fissure. Elle se désagrège au moment même où l'écriture n'est plus capable de garder son autonomie face à une Révolution qui décidément rend vains tous les efforts de quiconque tente de lui échapper, et illusoire tout espoir de s'établir dans un espace à l'écart et protégé.

Donner une conclusion à l'histoire d'une vie qui n'a pas encore touché à sa fin est l'une des difficultés inhérentes au genre autobiographique. En d'autres termes, un problème s'impose à celui qui écrit : comment faire converger je narrant et je narré? Notre auteur, rompu à l'art du conte, ne conclut pas ses Mémoires sur une scène pathétique comme l'aurait été celle d'un père lisant à ses enfants les différents épisodes de sa vie - scène analogue à celle qu'avait choisie Rousseau comme épilogue à ses Confessions. Il abandonne son histoire au beau milieu en cédant la parole au je qui écrit et qui est visiblement obsédé par les péripéties post-thermidoriennes. Les Mémoires avortent en déviant de leur objectif premier, et s'achèvent sur les espoirs - d'ailleurs déçus peu après, ce que Marmontel passe sous silence - que son appel à l'assemblée primaire du canton de Gaillon avait réveillés. Une écriture quotidienne digne d'un journal intime se substitue au récit - au sens formel du mot - qui se contente de rendre l'état présent brut et, par là, remet en doute l'autorité du je narrant. Marmontel n'a plus qu'à attendre son retour sur la scène publique et à espérer que celui-ci l'aide à reconstituer son identité, sa persona. En bref, la fin ouverte et tronquée des Mémoires met involontairement en relief l'impuissance de l'auteur à se placer en dehors de l'Histoire, et offre une preuve supplémentaire 
que le mouvement irrésistible de la Révolution a commencé à s'insinuer dans le cœur de la production littéraire.

Pour la corrélation entre otium et écriture, les Mémoires de Marmontel témoignent du basculement de l'auteur, d'abord décidé à recourir à la pratique de l'otium dans son sens le plus noble - à savoir trouver refuge et recul nécessaire dans l'écriture -, puis contraint de renoncer à ces méthodes qui ne tardent pas à succomber à l'impact de la Révolution, à un déferlement qui ne laisse plus de zones libres, ni d'espace à l'épanouissement personnel et qui condamne l'otium. On serait tenté d'en déduire que dans cet ouvrage les marques d'otium présentes dans les deux premiers tiers du texte sont en réalité le produit d'un acte d'écriture, qu'elles ont été mises en place a posteriori par un récit rétrospectif duquel émerge la figure souveraine du je narrant, nettement distinguée de celle du je narré, dominant l'univers fictionnel, qui plus est située au-delà de la réalité de la Révolution. En conséquence, ce que la lecture de Marmontel suggère est le contrepoint, tout au moins un changement de perspective, par rapport à ce que le présent volume a l'ambition de montrer : ce n'est plus une situation privilégiée - l'otium - qui déclenche l'écriture et qui implique une remise en question de la souveraineté du sujet-protagoniste, mais bel et bien l'acte d'écriture qui crée en quelque sorte pour l'écrivain une sensation d'otium qui affleure à même l'écriture, dans la narration d'un passé révolu et nettement séparé de la condition présente de l'auteur. C'est la narration qui comme par enchantement permet au je narrant non seulement d'oublier l'incertitude de son sort, mais encore de se retrouver dans une certaine souveraineté. Mais hélas, l'équilibre atteint est éphémère. Pour un contemporain de la Révolution française, écrire sur soi revient toujours à parler en tant que sujet historiquement défini : c'est le sujet traumatisé et entraîné par le mouvement de l'Histoire qui aura toujours le dernier mot.

Reste à prendre en considération un autre témoignage autobiographique que constituent les textes de Madame Roland réunis sous le titre Mémoires par son ami Bosc au temps du Directoire, et qui se caractérisent par leur grande hétérogénéité. Après son arrestation, Madame Roland entreprend la rédaction de ses mémoires, en premier lieu dans le but de défendre son mari - ex-ministre de l'Intérieur - des attaques injustes dont il est victime de la part des Montagnards. La citation placée en exergue : «Aujourd'hui sur le trône, demain dans les fers ${ }^{16}$ " s'applique à son mari, mais aussi implicitement à celle qui se dit prête à affronter un destin contraire en suivant les prescriptions du 
stoïcisme. Ensuite, elle cherche une autre façon de remplir ses longues heures d'inactivité. L'écriture reste son choix de prédilection, mais on constate un changement à partir du mois d'août : elle choisit de laisser aller sa plume à son gré et d'écrire simplement ce qui lui plaît. Comme l'a fait Marmontel et suivant probablement aussi les traces de Rousseau, elle se jette dans l'écriture pour prendre du recul mais sans bouder son plaisir. Le 9 août elle s'attelle à ses Mémoires particuliers.

Contrairement à Marmontel, Manon Roland ne cache pas que sa situation est à désespérer. Ses Mémoires s'ouvrent sur une description quelque peu pathétique :

Fille d'artiste, femme d'un savant devenu ministre et demeuré homme de bien, aujourd'hui prisonnière, destinée peut-être à une mort violente et inopinée, j'ai connu le bonheur et l'adversité, j'ai vu de près la gloire et subi l'injustice. (MMR, p. 305)

Elle englobe dans une seule phrase son origine sociale, sa surprenante carrière politique aux côtés de son mari, et son malheur présent qui laisse présager une fin funeste. Elle glisse très vite d'indications biographiques à une appréciation générale sur sa vie qui fait penser à l'épigraphe citée un peu plus haut. Tout en proposant fièrement sa vie comme objet d'étude au motif qu'elle est hors du commun grâce à une destinée que ni sa naissance dans un milieu modeste ni son sexe n’avaient laissé présager, elle change rapidement de direction et se limite à remonter au temps de sa jeunesse. Elle opte pour des formules au ton incantatoire telles que «Renouvelez-vous pour moi, moments tranquilles de ma douce adolescence!» ( $M M R$, p. 363) pour évoquer un passé qui désormais lui semble extrêmement lointain. Tantôt elle se prend à invoquer tous les saints pour que les images riantes et pleines de promesses de sa jeunesse se substituent au présent oppressant, tantôt elle en appelle au lecteur et le supplie de s'arrêter encore un moment "sur ces temps paisibles de saintes illusions auxquels [elle] aime encore à [se] reporter» $(M M R$, p. 345). Aux yeux de cette républicaine convaincue, la vie passée sous l'Ancien Régime est curieusement presque synonyme d'une vie vouée à l'otium, alors qu'une accumulation interminable de negotia où règnent l'inquiétude et l'injustice pèse sur les années de la Révolution. Plus elle se plonge dans l'évocation de cette "douceur de vivre" - selon la formule consacrée par Talleyrand -, plus elle trouve de réconfort dans ce retour à soi.

Le même mécanisme que chez Marmontel se met donc en place chez Madame Roland. Tant que l'évocation se poursuit, le récit des aventures du je narré donne le jour à la figure d'un je narrant qui assoit son autorité et adresse des commentaires de nature pédagogique à ses lecteurs. Quoique d'une manière différente de celle que l'on trouve chez Marmontel, dans le récit de 
Madame Roland s'intercalent pourtant des passages dans lesquels se manifeste le désarroi du je narré traumatisé par le tourbillon de la Révolution. Sous sa plume, se place dès le début à côté du je narré un je narrant double : l'un est traditionnel, il incarne l'autorité et demeure étroitement lié au je narré; l'autre est instantané pour ainsi dire, il est entraîné par la dynamique révolutionnaire et pour cette raison met en doute l'autorité supposée stable de son double. Alors que le je narrant instantané n'accède à l'espace d'otium qu'en s'anéantissant, le je narrant traditionnel ne semble être qu'un effet du récit rétrospectif. Par ailleurs, la coïncidence n'est peut-être pas fortuite : autant chez Madame Roland que chez Marmontel l'effet miraculeux de la narration ne se produit qu'à condition qu'ils évoquent leur passé sous l'Ancien Régime. Aux yeux des contemporains de la Révolution, l'Ancien Régime se présente en amont comme un univers enclin à l'otium. Ou bien est-ce le contraste apporté par une Révolution tout en action et en mouvement qui conférait ce charme à un monde d'ores et déjà révolu?

Comme chez Marmontel, le je narrant instantané finit par l'emporter. Madame Roland peine de plus en plus à évoquer le passé. Dos au mur, elle met fatalement un terme à son écrit parce qu'elle n'est plus à même de conduire sa plume "au milieu des horreurs qui déchirent [sa] patrie» (MMR, p. 520). Son geste suicidaire ne manque pas de pathétique. Mais elle ne cesse pas d'écrire pour autant. Elle s'adonne bien plutôt à une autre forme d'écriture, qui consiste à noter tout ce qui lui passe par la tête et ne vise donc plus à lier, à organiser les choses, mais qui en est réduite à capter les reflets furtifs d'une âme aux prises avec le mouvement irrésistible de la Révolution. Les Mémoires particuliers aboutissent à une sorte d'écriture éclatée qui a déjà retenu l'attention de la critique ${ }^{17}$. Cette écriture n'est pas seulement due aux circonstances; le besoin constant d'écrire, ressenti jusqu'au dernier instant - Madame Roland demandera du papier au pied de la guillotine - montre davantage que l'écriture, à mesure que le je narrant instantané l'emporte, est amenée à épouser le mouvement de la plume. On est tenté de conclure que l'écriture elle-même finit par être ressentie comme un mouvement qui entraîne et menace d'assujettir celui qui a pris la plume.

En conclusion, les auteurs mémorialistes de la Révolution héritent de la tradition latine renouvelée par Rousseau et projettent en aval la corrélation entre écriture et otium. Plus ils écrivent, plus ils se rendent compte que cet

I7. B. Didier définit les dernières pages des Mémoires de Madame Roland comme une «écriture disloquée» dans Écrire la révolution, I789-I799, ouvr. cité, p. 264. M. Cardy, de son côté, découvre dans les Mémoires de Marmontel «a kind of littérature éclatée», voir "Marmontel's Mémoires Revisited", art. cité, p. I83. 
héritage est remis en cause par la nouvelle façon de vivre l'Histoire, perçue comme un mouvement qui se déroule selon ses propres règles. Madame Roland et Marmontel se réfugient dans l'écriture, dans le récit de leur vie et pour quelque temps peuvent se réjouir d'y trouver du recul et une certaine assurance. L'héritage malgré tout ne peut perdurer qu'à condition qu'il se restreigne à l'univers clos du récit. Mais ni Madame Roland ni Marmontel n'auront la consolation de voir leur écriture et leur récit parvenir à contrer jusqu'au bout la pression révolutionnaire. Les mémoires mettent en évidence le fait que derrière le je narrant émerge un je qui vit et écrit au jour le jour. Alors que le je narrant, par l'acte même du récit, verse dans une sorte d'atemporalité tranquille, le je qui écrit est précipité dans le déroulement chaotique et rapide du temps historique. Par moments on peut certes l'oublier dans la matière du récit qui évolue, mais il finit toujours par revenir sur le devant de la scène et s'impose au détriment du récit. Ce je qui vit et écrit est livré aux caprices du mouvement révolutionnaire et devine que l'écriture elle-même en est pour ainsi dire contaminée. Les deux mémorialistes pressentent que l'écriture est en train de se transformer en un mouvement qui évolue selon ses propres lois : c'est la définition que Roland Barthes a donnée de l'écriture littéraire moderne ${ }^{18}$. Mais ils n'étaient pas encore en mesure de frayer le chemin que Chateaubriand prendra dans les années i 820 et de fixer comme lui leur je narrant dans l'outretombe, dans une sorte de mort anticipée qui libère l'auteur de toute casualité et l'élève au-dessus du tourbillon déclenché.

18. Voir à ce propos son essai «La mort de l'auteur», dans R. Barthes, Le Bruissement de la langue, Paris, Seuil, I984, p. 6I-67. 